\title{
The periplasmic thioredoxin SoxS plays a key role in activation in vivo of chemotrophic sulfur oxidation of Paracoccus pantotrophus
}

\author{
Grazyna Orawski, Frank Bardischewsky, Armin Quentmeier, \\ Dagmar Rother and Cornelius G. Friedrich \\ Lehrstuhl für Technische Mikrobiologie, Fachbereich Bio- und Chemieingenieurwesen, \\ Universität Dortmund, Emil-Figge-Strasse 66, D-44221 Dortmund, Germany
}

Correspondence

Cornelius G. Friedrich

cornelius.friedrich@udo.edu

Received 8 November 2006

Revised 18 December 2007

Accepted 22 December 2006

\begin{abstract}
The significance of the soxS gene product on chemotrophic sulfur oxidation of Paracoccus pantotrophus was investigated. The thioredoxin SoxS was purified, and the $\mathrm{N}$-terminal amino acid sequence identified SoxS as the soxS gene product. The wild-type formed thiosulfate-oxidizing activity and Sox proteins during mixotrophic growth with succinate plus thiosulfate, while there was no activity, and only traces of Sox proteins, under heterotrophic conditions. The homogenote mutant strain $\mathrm{GB} \Omega S$ is unable to express the soxSR genes, of which soxR encodes a transcriptional regulator. Strain $\mathrm{GB} \Omega S$ cultivated mixotrophically showed about $22 \%$ of the specific thiosulfate-dependent $\mathrm{O}_{2}$ uptake rate of the wild-type, and when cultivated heterotrophically it produced $35 \%$ activity. However, under both mixotrophic and heterotrophic conditions, strain $\mathrm{GB} \Omega S$ formed Sox proteins essential for sulfur oxidation in vitro at the same high level as the wild-type produced them during mixotrophic growth. Genetic complementation of strain $\mathrm{GB} \Omega S$ with soxS restored the activity upon mixotrophic and heterotrophic growth. Chemical complementation by reductants such as L-cysteine, DTT and tris(2-carboxyethyl)phosphine also restored the activity of strain $\mathrm{GB} \Omega S$ in the presence of chloramphenicol, which is an inhibitor of de novo protein synthesis. The data demonstrate that SoxS plays a key role in activation of the Sox enzyme system, and this suggests that SoxS is part of a novel type of redox control in P. pantotrophus.
\end{abstract}

\section{INTRODUCTION}

Reactions of the oxidative half of the global sulfur cycle are mediated by various aerobic lithotrophic and anaerobic phototrophic prokaryotes. Paracoccus pantotrophus is a Gram-negative, neutrophilic, facultatively chemolithoautotrophic bacterium that aerobically oxidizes thiosulfate, hydrogen sulfide and sulfur (Rainey et al., 1999; Robertson \& Kuenen, 1983). The gene region of $P$. pantotrophus encoding chemotrophic sulfur oxidation comprises 15 genes, namely soxSR and soxVWXYZABCDEFGH. Seven genes encode the proteins SoxYZ, SoxB, SoxCD and SoxXA. Together, these proteins catalyse hydrogen sulfide-, sulfur-, thiosulfate- and sulfite-dependent cytochrome $c$ reduction in vitro (Friedrich et al., 2001, 2005; Rother et al., 2001).

The soxSR genes are transcribed divergently to the other sox genes of the cluster. soxR encodes the repressor SoxR, which is a member of the ArsR family that binds to two intergenic regions (soxS-soxV and soxW-soxX) of the sox locus (Rother

Abbreviation: TCEP, tris(2-carboxyethyl)phosphine. et al., 2005), and soxS encodes the periplasmic thioredoxin SoxS (Rother et al., 2001).

The soxS gene is disrupted by the $\Omega$-kanamycin-resistanceencoding interposon Tn5 in the homogenote mutant $P$. pantotrophus $\mathrm{GB} \Omega S$, and this strain is also unable to express soxR. Strain GB $\Omega S$ has about $22 \%$ of the specific thiosulfatedependent $\mathrm{O}_{2}$ uptake rate of the wild-type level under mixotrophic growth conditions with succinate plus thiosulfate. Complementation of strain $\mathrm{GB} \Omega S$ with soxR restores repression of the specific thiosulfate-oxidizing activity under heterotrophic conditions, suggesting that SoxR is a repressor protein (Rother et al., 2005). The position of the soxS gene upstream of $\operatorname{soxR}$, and the low thiosulfate-oxidizing activity of $G B \Omega S$, seemed to suggest a function of the putative thioredoxin SoxS in the regulation of expression of chemotrophic sulfur oxidation.

In this study, we report the isolation of the periplasmic thioredoxin SoxS, and that it is encoded by the soxS gene. We demonstrate that a mutant unable to express soxS forms high levels of Sox proteins that are not active, and that SoxS is involved in activation of the thiosulfate-oxidizing enzyme 
system, and we exclude the involvement of SoxS in the expression of the sox genes.

\section{METHODS}

Bacterial strains and plasmids. Strains and plasmids used and constructed in this study are listed in Table 1. The DNA sequence of the sox gene region is accessible under GenBank/EMBL accession number X79242.

Media and growth conditions. Escherichia coli strains were cultivated in Luria-Bertani (LB) medium (Sambrook et al., 1989) at $37^{\circ} \mathrm{C}$. P. pantotrophus strains were cultivated under mixotrophic growth conditions in mineral medium, initial pH 7.2, with $20 \mathrm{mM}$ thiosulfate plus $20 \mathrm{mM}$ disodium succinate (Bardischewsky \& Friedrich, 2001), unless otherwise stated. Heterotrophic growth was performed in mineral medium at an initial $\mathrm{pH}$ of 6.8 , with $20 \mathrm{mM}$ succinate at $30^{\circ} \mathrm{C}$. L-Cysteine, DTT and tris(2-carboxyethyl)phosphine (TCEP) were added as specified in the text. Antibiotics were added where appropriate (for E. coli: $100 \mu \mathrm{g}$ ampicillin $\mathrm{ml}^{-1}, 50 \mu \mathrm{g}$ chloramphenicol $\mathrm{ml}^{-1}$, and $50 \mu \mathrm{g}$ kanamycin $\mathrm{ml}^{-1}$; for P. pantotrophus: $300 \mu \mathrm{g}$ kanamycin $\mathrm{ml}^{-1}, 10 \mu \mathrm{g}$ chloramphenicol $\mathrm{ml}^{-1}$, and $40 \mu \mathrm{g}$ rifampicin $\mathrm{ml}^{-1}$ ).

DNA techniques. Standard DNA techniques were applied (Sambrook et al., 1989). Plasmids were prepared in small scale from E. coli, as described by Kieser (1984). For DNA sequencing, plasmid DNA was prepared with the Qiagen Plasmid Midi Kit (Qiagen). Starting from the known sequence, 2674 bp of pOG7 were further sequenced downstream of soxR. DNA sequencing was done by Invitec. Restriction enzymes, T4 DNA ligase, alkaline phosphatase and Klenow polymerase were obtained from Fermentas, Promega or Roche, and used as recommended by the manufacturer. Agarose gel electrophoresis was done in TAE (Sambrook et al., 1989). DNA fragments were eluted from agarose gels using the QIAquick gel extraction kit (Qiagen).

Construction of pRIsoxR, pRIsoxS and pRIsoxSR. The soxS gene was disrupted by inserting an $\Omega$-kanamycin interposon by gene replacement, yielding the homogenote mutant $P$. pantotrophus $\mathrm{GB} \Omega S$, as described by Rother et al. (2005). Strain GB $\Omega S$ was complemented with soxR by plasmid pRIsoxR (Rother et al., 2005). Plasmid pRIsoxS was constructed for complementation of strain
GB $\Omega$ S with soxS. Plasmid pOG7 (Table 1) was cut with BamHI and $K p n I$, and the generated $608 \mathrm{bp}$ fragment containing soxS was cloned into pRI1, resulting in the plasmid pRIsoxS. For complementation with SoxR and SoxS, pOG7 was cut with KpnI and SalI. The generated $3192 \mathrm{bp}$ fragment containing soxSR was cloned into pRI1, resulting in the plasmid pRIsoxSR. E. coli strains were transformed as described by Chung et al. (1989). E. coli S17-1 was used to mobilize the plasmids pRIsoxR, pRIsoxS and pRIsoxSR into P. pantotrophus GB17 and GBRS (Simon et al., 1983).

Preparation of cell fractions. Periplasmic proteins were extracted from the cells by osmotic shock according to the QIAexpressionist (2nd edn, Protocol 4; Qiagen). Tris/ $\mathrm{HCl}$ was added to the extract to give a final concentration of $25 \mathrm{mM}$.

Cell-free extracts were prepared by passing cell suspensions $(0.1 \mathrm{~g}$ dry cell weight $\mathrm{ml}^{-1}$ ) twice through a French pressure cell at $150 \mathrm{MPa}$, and subjecting the extracts to differential centrifugation. The $200000 \mathrm{~g}$ supernatant was used for ammonium sulfate fractionation. Proteins precipitating between 44 and $65 \%$ ammonium sulfate saturation were dialysed against $25 \mathrm{mM}$ sodium/potassium phosphate buffer, $\mathrm{pH} 6.5$, and designated as the A65 fraction (Friedrich et al., 2000).

Purification of the thioredoxin SoxS. SoxS was identified by antibodies raised against the immunogenic oligopeptides OP-S with the amino acid sequences LQMRDPLPPGLELAR and DVESGRLEGYPGED, as deduced from the soxS nucleotide sequence. SoxS was purified from the A65 fraction of cells, which were cultivated mixotrophically with succinate plus thiosulfate, using similar methods to those described for the Sox proteins that constitute the Sox enzyme system (Rother et al., 2001). The protein was subjected to chromatography on Q-Sepharose Fast Flow, and eluted in the $0.10 \mathrm{M}$ sodium chloride fraction of the respective step gradient. The SoxS-containing fractions were identified by Western blot analysis (Towbin et al., 1979), and then combined, concentrated by ultrafiltration and subjected to gel-filtration chromatography on Sephacryl S200 HR. For further purification, SoxS-containing fractions were subjected to chromatography on Phenyl Sopharose High Performance, which is a hydrophobic interaction matrix. This step yielded a partially purified preparation of SoxS that was analysed by immunoblotting.

Analytical procedures. Denatured proteins were separated by SDS-PAGE according to Laemmli (1970). Proteins were stained

Table 1. Bacterial strains and plasmids

\begin{tabular}{|c|c|c|}
\hline Strain or plasmid & Relevant genotype or phenotype & Reference or source \\
\hline \multicolumn{3}{|l|}{ E. coli } \\
\hline JM 109 & $\begin{array}{l}\text { recA1 supE44 endA1 hsdR17 gyrA96 relA1 thi } \Delta(\text { lac-proAB }) \\
\mathrm{F}^{\prime}\left[\operatorname{traD} 36 \text { proAB }{ }^{+} \text {lacl }{ }^{\mathrm{q}} \text { lacZ } \Delta \mathrm{M} 15\right]\end{array}$ & Yanisch-Perron et al. (1985) \\
\hline S17-1 & recA pro thi hsdS RP4-tra-functions supE44 & Simon et al. (1983) \\
\hline \multicolumn{3}{|l|}{ P. pantotrophus } \\
\hline GB17 & Sox $^{+}$ & Rainey et al. (1999) \\
\hline $\mathrm{GB} \Omega \mathrm{S}$ & $\begin{array}{l}\text { Homogenote mutant of GB17 with } \Omega \text {-kanamycin-resistance cassette } \\
\text { in soxS }\left(\operatorname{sox} S:: \Omega-\mathrm{Km}^{\mathrm{r}}\right)\end{array}$ & Rother et al. (2005) \\
\hline \multicolumn{3}{|l|}{ Plasmids } \\
\hline pOG7 & $4 \mathrm{~kb} E c o \mathrm{RI}-K p n \mathrm{I}$ fragment soxR-orf $\mathrm{V}^{\prime}$ in pUC19 & Rother et al. (2005) \\
\hline pRI1 & $\mathrm{Cm}^{\mathrm{r}}, \mathrm{Sm}^{\mathrm{r}}$ & Pfitzner et al. (1998) \\
\hline pRIsoxR & 1942 bp SmaI fragment of pOG7 containing sox $R^{-}$soxS in pRI1 & Rother et al. (2005) \\
\hline pRIsoxS & 608 bp KpnI-BamHI fragment of pOG7 containing soxS in pRI1 & This study \\
\hline $\mathrm{pRI}$ soxSR & 3192 bp KpnI-Sall fragment of pOG7 containing soxSR in pRI1 & This study \\
\hline
\end{tabular}


with Coomassie blue, as described by Weber et al. (1972). Protein from cell-free extracts was determined by the method of Bradford (1976).

Immunoblots (Western blots) (Towbin et al., 1979) were performed according to the 'semi-dry' procedure, using the multiphor electrophoresis system (Pharmacia) and antibodies raised in rabbits, at the facilities of Eurogentec. SoxYZ antigens were detected with polyclonal antibodies raised against homogeneous SoxYZ. SoxCD antigens were detected with polyclonal antibodies raised against homogeneous SoxCD. Antibodies raised against the cytochrome complex SoxXA of $P$. pantotrophus were used to detect SoxXA. SoxB antigens were detected with antibodies against SoxB antigens expressed in E. coli (Friedrich et al., 2000).

The N-terminal amino acid sequence was determined from purified SoxS, which was separated by SDS-PAGE, and transferred to ProBlot membranes. The major protein band of $11 \mathrm{kDa}$ was cut out of the membrane, and subjected to automated Edman degradation using the protein sequencer system model 494A/190A (Applied Biosystems), as detailed previously (Fischer et al., 1996). The signal peptide cleavage site was predicted by the PSORT program package (Nakai \& Kaneshisa, 1991).

The PHYLIP package (Felsenstein, 1989) was used to calculate protein distance matrices, and to construct phylogenetic trees. For settings, standard options were used. Trees were drawn using TREEVIEW, version 1.6.6. (Page, 1996).

Enzyme assays. The thiosulfate oxidation rate of whole cells was determined with an oxygen electrode (Rank Brothers). The assay $(3 \mathrm{ml})$ contained $50 \mu \mathrm{l}$ cell suspension $\left(\mathrm{OD}_{436} 30\right.$, equivalent to $150 \mu \mathrm{g}$ protein) and $100 \mu \mathrm{mol}$ sodium/potassium phosphate buffer ( $\mathrm{pH}$ 8.0). Reactions were started with $30 \mu \mathrm{l} 0.20 \mathrm{M}$ sodium thiosulfate. The specific thiosulfate-dependent $\mathrm{O}_{2}$ uptake rate was measured as $\mu \mathrm{mol} \mathrm{O}_{2}$ consumed $\min ^{-1}$ ( $\mathrm{mg}$ protein) ${ }^{-1}$.

\section{RESULTS AND DISCUSSION}

\section{Expression of soxS}

The soxSR genes are oriented divergently to the other genes of the sox cluster. Expression of soxS was analysed by immunoblot analysis of periplasmic extracts using the SoxSspecific OP-S-antibodies. SoxS antigens were detected in periplasmic extracts of wild-type cells cultivated mixotrophically with thiosulfate, while no SoxS antigens were detected from heterotrophically grown cells (Fig. 1a). As expected, no SoxS antigens were detected from the A65 fraction of extracts of the homogenate mutant strain $\mathrm{GB} \Omega S$ (Fig. 1b) harbouring a soxS:: $\Omega$-disruption (Rother et al., 2005). To complement strain GB $\Omega S$ with soxS or soxSR, the two plasmids pRIsoxS and pRIsoxSR (Table 1) were constructed, and conjugated into strain $\mathrm{GB} \Omega S$. In both strains $\mathrm{GB} \Omega \mathrm{S}$ (pRIsoxS) and GB $\Omega \mathrm{S}$ (pRIsoxSR), SoxS antigens were present after mixotrophic growth (Fig. 1b). In periplasmic extracts, the signal of SoxS antigens was stronger, as observed from the A65 fraction of extracts of the wild-type GB17 that indicated the selectivity of the procedure. The faint signal of SoxS in the A65 fraction of strain GB17 indicated the low concentration of SoxS in vivo, and the stronger signals observed from the respective extracts of the complemented strains of GB $\Omega$ S showed the (a)

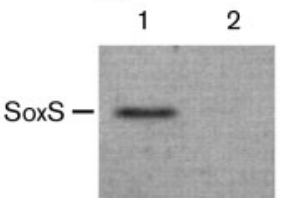

(b)

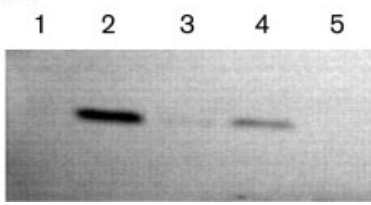

Fig. 1. Detection of SoxS in strains of $P$. pantotrophus by Western blot analysis. (a) Periplasmic fractions (30 $\mu \mathrm{g}$ protein per well) of GB17 after mixotrophic cultivation with thiosulfate (lane 1), and after heterotrophic cultivation (lane 2). (b) A65 fractions $(20 \mu \mathrm{g}$ protein per well) of cells grown mixotrophically with thiosulfate. Lanes: 1, GB $\Omega S$; 2, GB $\Omega S(p R I s o x S) ; 3$, GB17; 4, GB $\Omega S(p R \mid s o x S R) ; 5$, GB $\Omega S(p R I s o x R)$.

higher rate of expression of the soxS gene from the plasmids pRIsoxS and pRIsoxSR (Fig. 1b).

\section{Purification of the thioredoxin SoxS}

SoxS, as deduced from the soxS nucleotide sequence, consists of 130 aa, with a molecular mass of $14467 \mathrm{Da}$. The mature SoxS is predicted to contain 99 aa, with a molecular mass of $11077 \mathrm{Da}$. SoxS was purified as detailed in Methods, and the OP-S antibodies identified an $11 \mathrm{kDa}$ protein (data not shown). Ten amino acids of the $\mathrm{N}$ terminus of SoxS were analysed to be AELRLLMSEQ. This sequence was identical to that deduced from soxS. Also, the $\mathrm{N}$-terminus Ala-32 was identical to that predicted for the signal peptide cleavage site. These data identified soxS as encoding the periplasmic protein SoxS, and this result matched well with the periplasmic location of SoxS antigens (Fig. 1a).

\section{Sequence analysis of SoxS}

The low molecular mass (11077 Da) and the CXXC motif were diagnostic for thioredoxins. Paracoccus denitrificans 1222 is the closest relative of $P$. pantotrophus GB17 (Rainey et al., 1999), which was previously classified as $P$. denitrificans GB17 (Ludwig et al., 1993). From the genome sequence of Paracoccus denitrificans 1222 (accession no. AAIT00000000), eight proteins with characteristics of thioredoxins were identified. Of these, six were predicted to be located in the periplasm, and two in the cytoplasm (Fig. 2), all being rather diverse, and suggesting different functions and partner proteins. The amino acid sequences of thioredoxins predicted from the genomes of other bacteria were aligned together with those for which redox function has been shown. The alignment revealed that SoxShomologous thioredoxins clustered in a distinct group differentiated from the other thioredoxins (data not shown). The BLAST search demonstrated that proteins highly homologous to SoxS were exclusively present in sulfur-oxidizing chemotrophic and phototrophic Alphaproteobacteria, which harbour sox gene clusters (data not shown). These data revealed that thioredoxins of the SoxS-type were distinct 


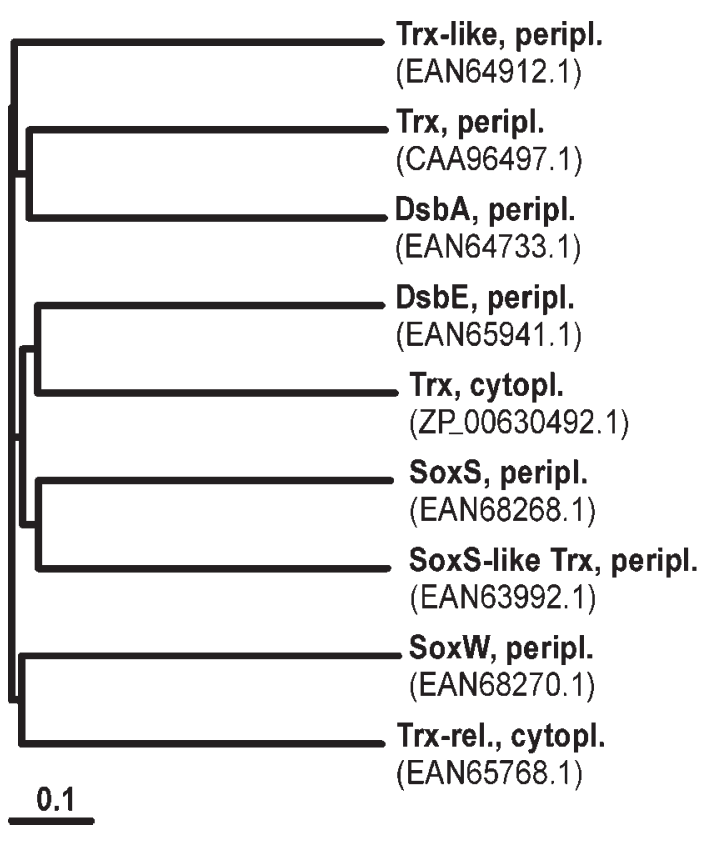

Fig. 2. Phylogenetic tree of putative thioredoxins of $P$. denitrificans PD1222 and the relationship to SoxS of $P$. pantotrophus. The tree was constructed with the software specified in Methods. The accession numbers of the proteins are given in parentheses.

from other periplasmic thioredoxins, and from those located in the cytoplasm or attached to the cytoplasmic membrane. This analysis suggests a specific function for SoxS thioredoxins in chemotrophic sulfur metabolism.

\section{Analysis of the SoxS function}

SoxR was shown to bind to the intergenic regions soxS-soxV and $\operatorname{soxW}-\operatorname{soxX}$, and it was identified as a repressor of the transcription of the respective sox genes (Rother et al., 2005). To analyse whether SoxS was involved in the regulation of the transcription of the sox genes, immunoblot analysis was performed to detect the Sox-relevant proteins in cell-free extracts of strains GB17, GB $\Omega S, G B \Omega S$ (pRIsoxR), $\mathrm{GB} \Omega \mathrm{S}(\mathrm{pRI}$ soxS) and GB$\Omega \mathrm{S}$ (pRIsoxSR). The proteins SoxXA, SoxYZ, SoxB and SoxCD were present at high concentrations in cell-free extracts of the wild-type GB17 grown mixotrophically with thiosulfate, while only traces of the proteins were detected in wild-type cells cultivated heterotrophically (Fig. 3). Mixotrophically, as well as heterotrophically, cultivated cells of strain $\mathrm{GB} \Omega S$ and $\mathrm{GB} \Omega S$ (pRIsoxS) formed the Sox proteins at the same level as the wild-type when it was cultivated under mixotrophic conditions. However, expression of soxR in trans by plasmids pRIsoxR and pRIsoxSR restored the wild-type phenotype in the complemented strains of GB $\Omega$ S (Fig. 3). Therefore SoxR, but not SoxS, was involved in repression of the transcription of the sox genes under heterotrophic growth conditions. (a)
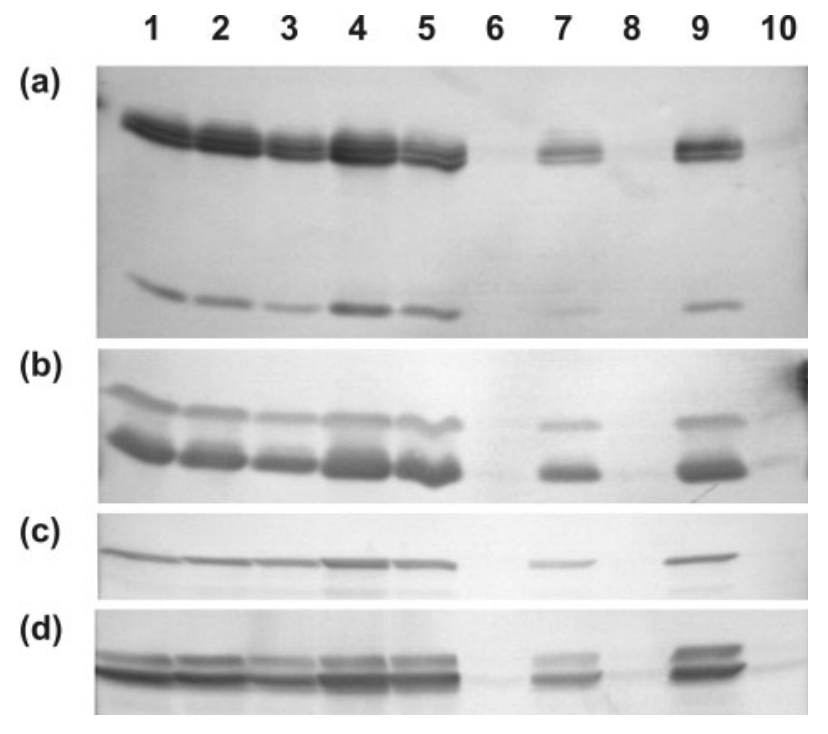

Fig. 3. Sox protein formation in strains of $P$. pantotrophus. SoxXA (a), SoxYZ (b), SoxB (c) and SoxCD (d) were detected by Western blot in $A 65$ fractions $(20 \mu \mathrm{g}$ protein per well) from cells grown mixotrophically with thiosulfate (lanes 1-5), and heterotrophically (lanes 6-10). Lanes: 1 and 6, GB17; 2 and 7, GB $\Omega$; 3 and 8, GB $\Omega S(p R I s o x R) ; 4$ and 9, GB $\Omega S(p R I s o x S)$; 5 and $10, \mathrm{~GB} \Omega \mathrm{S}(\mathrm{pRI}$ soxSR).

After mixotrophic cultivation, the specific thiosulfateoxidizing activity of whole cells of strain $G B \Omega S$ was about $22 \%$ of that of the wild-type. Moreover, trans-complementation of strain GB $\Omega S$ with soxR had no effect on the activity of whole cells (Rother et al., 2005).

To determine if SoxS affected the activity of the Sox enzyme system in vivo, the specific thiosulfate-oxidizing activity was determined from mixotrophically cultivated cells of the wild-type GB17, and strains GB $\Omega$, GB $\Omega$ (pRIsoxR),

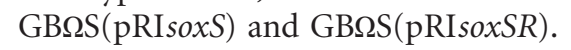

As expected, under mixotrophic growth conditions, the wild-type formed a high specific thiosulfate-oxidizing activity of $0.57 \mu \mathrm{mol} \mathrm{O} \mathrm{O}_{2} \min ^{-1}(\mathrm{mg} \text { protein })^{-1}$. Strain GB $\Omega S$ exhibited a low specific thiosulfate-oxidizing activity of $0.13 \mu \mathrm{mol} \mathrm{O}_{2} \min ^{-1}$ (mg protein) ${ }^{-1}$ (Table 2), while the Sox proteins were present at the same concentration as in the wild-type (Fig. 3).

Complementation of strain GB $\Omega S$ with the soxS gene resulted in a full restoration of the specific thiosulfateoxidizing activity, and complementation to soxSR increased the specific thiosulfate-oxidizing activity of whole cells to about $48 \%$, as compared with the wild-type. However, expression of soxR alone had no effect on the specific thiosulfate-dependent $\mathrm{O}_{2}$ uptake rate (data not shown). This result strongly suggests that SoxS plays a key role in activation of one or more proteins of the Sox enzyme system of $P$. pantotrophus. 
Table 2. Activation of thiosulfate oxidation in strain $\mathrm{GB} \Omega S$ by reductants

Cells were cultivated mixotrophically with succinate plus thiosulfate, and samples were taken after 30 min aerobic incubation at $30^{\circ} \mathrm{C}$. ND, Not determined.

\begin{tabular}{|c|c|c|c|}
\hline \multirow[t]{2}{*}{ Reductant } & \multirow[t]{2}{*}{ Concn $(\mathbf{m M})$} & \multicolumn{2}{|c|}{$\begin{array}{l}\text { Specific thiosulfate-dependent } \mathrm{O}_{2} \\
\text { uptake rate }\left[\mu \mathrm{mol} \mathrm{O}_{2} \min ^{-1}(\mathrm{mg} \text { protein })^{-1}\right]\end{array}$} \\
\hline & & Wild-type GB17 & $\mathrm{GB} \Omega S$ \\
\hline None & - & 0.57 & 0.13 \\
\hline L-Cysteine & 5 & 0.61 & 0.38 \\
\hline L-Cysteine & 1 & 0.52 & 0.21 \\
\hline DTT & 1 & 0.61 & 0.46 \\
\hline $\mathrm{DTT}^{\star}$ & 1 & ND & 0.46 \\
\hline $\mathrm{DTT} \dagger$ & 1 & ND & 0.61 \\
\hline TCEP & 1 & 0.54 & 0.29 \\
\hline
\end{tabular}

${ }^{*}$ Chloramphenicol $\left(10 \mu \mathrm{g} \mathrm{ml}^{-1}\right)$ was also added.

$\dagger$ Rifampicin $\left(40 \mu \mathrm{g} \mathrm{ml}^{-1}\right)$ was also added.

\section{Reductants activate the Sox enzyme system in vivo}

The membrane protein SoxV is essential for the function of the Sox enzyme system in $P$. pantotrophus and the phototroph Rhodovulum sulfidophilum (Bardischewsky et al., 2006; Appia-Ayme \& Berks, 2002). However, the function of SoxV can be chemically compensated by addition of the reductant DTT, resulting in the transient restoration of the specific thiosulfate-oxidizing activity under aerobic conditions (Bardischewsky et al., 2006). SoxV of $P$. pantotrophus is a paralogue of the DsbD/CcdA family, which also transports electrons from the cytoplasm to the periplasm. However, DsbD/CcdA proteins exclusively function in reduction of apocytochromes to enable addition of the haem moiety. Also, the phenotypes of some $d s b A B$ and $c c d A / d s b D$-defective strains can be restored by addition of thiol compounds to the medium (Bardwell et al., 1993; Sambongi \& Ferguson, 1994). Therefore, the effect of reductants on the specific thiosulfate-oxidizing activity of strain GB $\Omega S$ when cultivated mixotrophically with succinate plus thiosulfate was examined.

Addition of $1 \mathrm{mM}$ L-cysteine to the medium led to a 1.6-fold increase in specific thiosulfate-oxidizing activity of strain $\mathrm{GB} \Omega S$, but no increase was seen for the wild-type. The activity increase depended on the concentration of Lcysteine, and $5 \mathrm{mM}$ L-cysteine increased the activity threefold (Table 2). The increase occurred within $30 \mathrm{~min}$, and was maintained at the high level for at least $3 \mathrm{~h}$ (data not shown). Also, addition of DTT (1 mM) and the non-sulfur reductant TCEP ( $1 \mathrm{mM})$ increased the specific thiosulfateoxidizing activity of whole cells. Inclusion of chloramphenicol, an inhibitor of translation, or rifampicin, an inhibitor of transcription, prior to the addition of DTT, did not affect the increase in activity (Table 2). From these studies, and the rapid effect of the various reductants, it was concluded that one or more Sox proteins present in strain $G B \Omega S$ were activated by reduction, while de novo protein synthesis was not involved in the observed increase in specific thiosulfateoxidizing activity.

\section{The Sox enzyme system is active in extracts of strain GB $\Omega S$}

Immunoblot analysis of strain $G B \Omega S$ revealed a high concentration of the Sox proteins (Fig. 3). However, strain $\mathrm{GB} \Omega S$ exhibited a low in vivo specific thiosulfate-dependent $\mathrm{O}_{2}$-uptake rate (Table 2). For high activity, strain $\mathrm{GB} \Omega S$ required either genetic complementation to produce SoxS, or chemical complementation by reductants (Table 2 ). The in vitro specific thiosulfate-dependent cytochrome $c$ reduction rate, as examined from the ammonium sulfate fraction of the cell-free extract of strain $G B \Omega S$, was $2.2 \mathrm{mU}$

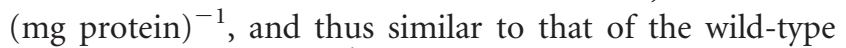
[1.9 $\mathrm{mU}$ (mg protein $\left.)^{-1}\right]$. From these data, SoxS appears to be required for the activation of one or more proteins of the specific thiosulfate-oxidizing enzyme system in vivo. The data do not suggest that SoxS is involved in the oxidation mechanism of thiosulfate, since the reconstituted Sox enzyme system functioned well without SoxS. This was evident from the in vitro studies of strain $\mathrm{GB} \Omega S$ specified above, and from biochemical studies (Rother et al., 2001). The membrane-bound protein SoxV is specifically required for sulfur oxidation in vivo, while the periplasmic thioredoxin SoxW is not. Therefore, one partner of SoxS might be SoxV, while the other partner is very likely to be at least one of the Sox proteins that constitute the Sox enzyme system. Future studies using sitedirected mutagenesis will aim to identify the redox partners of SoxS. 


\section{REFERENCES}

Appia-Ayme, C. \& Berks, B. C. (2002). SoxV, an orthologue of the CcdA disulfide transporter, is involved in thiosulfate oxidation in Rhodovulum sulfidophilum and reduces the periplasmic thioredoxin SoxW. Biochem Biophys Res Commun 296, 737-741.

Bardischewsky, F. \& Friedrich, C. G. (2001). The $s h x V W$ locus is essential for oxidation of inorganic sulfur and molecular hydrogen by Paracoccus pantotrophus GB17: a novel function in lithotrophy. FEMS Microbiol Lett 202, 215-220.

Bardischewsky, F., Fischer, J., Höller, B. \& Friedrich, C. G. (2006). SoxV transfers electrons to the periplasm of Paracoccus pantotrophus - an essential reaction for chemotrophic sulfur oxidation. Microbiology 152, 465-472.

Bardwell, J. C., Lee, J.-O., Jander, G., Martin, N., Belin, D. \& Beckwith, J. (1993). A pathway for disulphide bond formation in vivo. Proc Natl Acad Sci U S A 90, 1038-1042.

Bradford, M. M. (1976). A rapid and sensitive method for the quantitation of microgram quantities of protein utilizing the principle of protein-dye binding. Anal Biochem 72, 248-254.

Chung, C. T., Niemela, S. L. \& Miller, R. H. (1989). One-step preparation of competent Escherichia coli: transformation and storage of bacterial cells in the same solution. Proc Natl Acad Sci U S A 86, 2172-2175.

Felsenstein, J. (1989). PHYLIP - phylogeny inference package (version 3.2). Cladistics 5, 164-166.

Fischer, J., Quentmeier, A., Kostka, S., Kraft, R. \& Friedrich, C. G. (1996). Purification and characterization of the hydrogenase from Thiobacillus ferrooxidans. Arch Microbiol 165, 289-296.

Friedrich, C. G., Quentmeier, A., Bardischewsky, F., Rother, D., Kraft, R., Kostka, S. \& Prinz, H. (2000). Novel genes coding for lithotrophic sulfur oxidation of Paracoccus pantotrophus GB17. J Bacteriol 182, 4677-4687.

Friedrich, C. G., Rother, D., Bardischewsky, F., Quentmeier, A. \& Fischer, J. (2001). Oxidation of reduced inorganic sulfur compounds by bacteria: emergence of a common mechanism? Appl Environ Microbiol 67, 2873-2882.

Friedrich, C. G., Bardischewsky, F., Rother, D., Quentmeier, A. \& Fischer, J. (2005). Prokaryotic sulfur oxidation. Curr Opin Microbiol 8, 253-259.

Kieser, T. (1984). Factors affecting the isolation of ccc DNA from Streptomyces lividans and Escherichia coli. Plasmid 12, 19-36.

Laemmli, U. K. (1970). Cleavage of structural proteins during the assembly of the head of bacteriophage T4. Nature 227, 680-685.

Ludwig, W., Mittenhuber, G. \& Friedrich, C. G. (1993). Transfer of Thiosphaera pantotropha to Paracoccus denitrificans. Int J Syst Bacteriol 43, 363-367.
Nakai, K. \& Kaneshisa, M. (1991). Expert system for predicting protein localization sites in Gram-negative bacteria. Proteins 11, 95-110.

Page, R. D. M. (1996). TREEVIEW: an application to display phylogenetic trees on personal computers. Comput Applic Biosci 12, 357-358.

Pfitzner, U., Odenwald, A., Ostermann, T., Weingard, L., Ludwig, B. \& Richter, O. M. (1998). Cytochrome $c$ oxidase (heme $a a_{3}$ ) from Paracoccus denitrificans: analysis of mutations in putative proton channels of subunit I. Bioenerg Biomembr 30, 89-97.

Rainey, F. A., Kelly, D. P., Stackebrandt, E., Burghardt, J., Hiraishi, A., Katayama, Y. \& Wood, A. P. (1999). A re-evaluation of the taxonomy of Paracoccus denitrificans and a proposal for the combination Paracoccus pantotrophus comb. nov. Int J Syst Bacteriol 49, 645-651.

Robertson, L. A. \& Kuenen, J. G. (1983). Thiosphaera pantotropha gen. nov. sp. nov., a facultatively anaerobic, facultative autotrophic sulphur bacterium. J Gen Microbiol 129, 2847-2855.

Rother, D., Henrich, H.-J., Quentmeier, A., Bardischewsky, F. \& Friedrich, C. G. (2001). Novel genes of the sox gene cluster, mutagenesis of the flavoprotein SoxF, and evidence for a general sulfur oxidizing system in Paracoccus pantotrophus GB17. J Bacteriol 183, 4499-4508.

Rother, D., Orawski, G., Bardischewsky, F. \& Friedrich, C. G. (2005). SoxRS-mediated regulation of chemotrophic sulfur oxidation in Paracoccus pantotrophus. Microbiology 151, 1707-1716.

Sambongi, Y. \& Ferguson, S. J. (1994). Specific thiol compounds complement deficiency in $c$-type cytochrome biogenesis in Escherichia coli carrying a mutation in a membrane bound disulphide isomerase-like protein. FEBS Lett 353, 235-238.

Sambrook, J., Maniatis, T. \& Fritsch, E. F. (1989). Molecular Cloning: a Laboratory Manual, 2nd edn. Cold Spring Harbor, NY: Cold Spring Harbor Laboratory.

Simon, R., Priefer, U. \& Pühler, A. (1983). A broad host range mobilization system for in vivo genetic engineering: transposon mutagenesis in Gram negative bacteria. Bio/Technology 1, 784-790.

Towbin, H., Staehelin, T. \& Gordon, J. (1979). Electrophoretic transfer of proteins from polyacrylamide gels to nitrocellulose sheets: procedure and some applications. Proc Natl Acad Sci U S A 76, 6762-6766.

Weber, K., Pringle, J. R. \& Osborn, M. (1972). Measurement of molecular weights by electrophoresis on SDS-acrylamide gel. Methods Enzymol 26, 3-27.

Yanisch-Perron, C., Vieira, J. \& Messing, J. (1985). Improved M13 phage cloning vectors and host strains: nucleotide sequences of the M13mp18 and pUC19 vectors. Gene 33, 103-119.

Edited by: H. L. Drake 